\title{
ENTORNOS 3D PARA EL MODELADO DEL TERRENO
}

\section{D ENVIRONMENT FOR} LANDSCAPE MODELING

\author{
Carlos Carbonell Carrera \\ ccarbone@ull.edu.es
}

Stephany Hess Medler sthsess@ull.es

\section{Universidad de La Laguna, España}

https://doi.org/10.25145/b.innovaull.2019.019

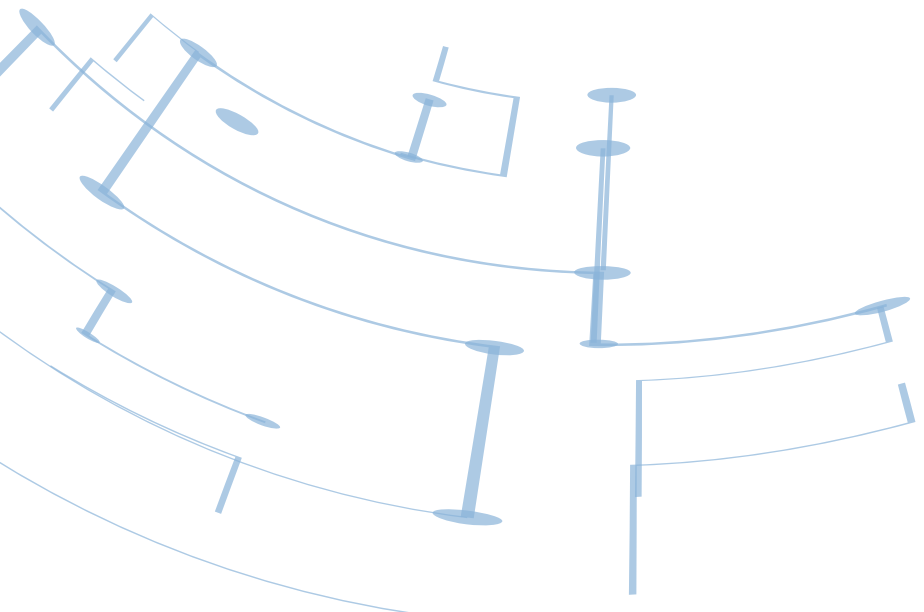




\section{RESUMEN}

En la educación de ciencias relacionadas con la representación de la superficie se tiende hacia un enfoque de enseñanza práctico, que implica por parte de profesores y alumnos de formación específica en Sistemas de Información Geográfica SIG y / o geotecnologías. Sin embargo, en las primeras etapas de la educación geoespacial, estas tareas se pueden realizar con tecnologías que no requieran profundos conocimientos de SIG. La presente investigación muestra los resultados de un taller realizado con estudiantes de ingeniería en un entorno de software de modelado 3D fácil de usar. Para medir el impacto de esta experiencia los participantes completaron un cuestionario de modelado 3D. Resultados por encima de 3 en una escala de Likert (1-5) confirman la utilidad de este enfoque de enseñanza utilizando tecnologías 3D fáciles de usar para desarrollar el pensamiento geoespacial.

PALABRAS CLAVE: interpretación del relieve; Skethc Up; modelado 3D.

\section{ABSTRACT}

The science education related to the representation of the Earth's surface tends towards a practical teaching approach, which involves teachers and students of specific training in Geographic Information Systems and / or geotechnologies. However, in the early stages of geospatial education, these tasks can be done with technologies that do not require deep knowledge of GIS. This research shows the results of a workshop conducted with engineering students in an easy-to-use 3D modeling software environment. To measure the impact of this experience, the participants completed a 3D modeling questionnaire. Results above 3 on a Likert scale (1-5) confirm the usefulness of this teaching approach using easy-to-use 3D technologies to develop geospatial thinking.

KEYWORDS: relief interpretation, 3D modeling, Sketch Up.

\section{INRODUCCIÓN}

Las competencias espaciales figuran como competencias a adquirir en las titulaciones de Grado y Máster del Espacio Europeo de Educación Superior (Linn y Petersen, 1985; Maier, 1998, Hegarty et al. 2006; Carbonell et al. 2011). En el ámbito de desarrollo de estas competencias, se han llevado a cabo diversos proyectos de innovación en la Universidad de La Laguna:

2010-11: Análisis de dispositivos de pantalla táctil como herramienta de innovación educativa en la docencia de ciencias geográficas: búsqueda y recuperación de información georeferenciada en infraestructuras de datos espaciales en soporte web.

2011 -12: Tablet 3D: taller de mejora de las relaciones espaciales sobre iPad. 2013-14: Realidad aumentada georeferenciada como herramienta para la visualización tridimensional de modelos digitales del terreno. 
2014-15: Inserción de mundos reales en el videojuego Minecraft para su uso educacional.

2015-16: La impresión 3D de modelos digitales de terreno para la mejora de las competencias espaciales y la interpretación del relieve cartográfico.

2016-17: Geomática y Realidad Virtual.

En la docencia de asignaturas relacionadas con la interpretación de la topografía del terreno se observan unas debilidades en el proceso enseñanza-aprendizaje propias de la dificultad que la abstracción tridimensional precisa del alumno para la comprensión del relieve: al alumno le cuesta interpretar el terreno. Es necesario el fomento de actividades que permitan desarrollar la capacidad de intepretación del relieve de los estudiantes de grados en los que se trabaja con mapas, planos e información georeferenciada.

\section{LA INTERPRETACIÓN DEL RELIEVE}

La interpretación de terrenos partiendo de mapas y/o planos en los que el relieve está representado a través de técnicas cartográficas tradicionales (curvado, sombreado cenital u oblicuo, tintas hipsométricas...) es compleja para el estudiante: no es una materia con la que suela estar familiarizado, y en asignaturas cuatrimestrales hay poco tiempo para desarrollar estos conceptos (Carbonell y Bermejo, 2017). La modelización digital de terrenos se ha venido haciendo a través de metodologías CAD que permiten la visualización de un modelo digital de terreno en tres dimensiones en la pantalla del ordenador. En la actividad desarrollada en este artíoculo lo que se pretende es que estudiante sea capaz de generar un modelo digital de terreno, de ser capaz de modelar un entorno paisajístico en el que existan una serie de elementos geomorfológicos previamente establecidos, empleando herramientas de modelado 3D digital de bajo coste y fáciles de utilizar.

Los objetivos son ayudar al alumno en la comprensión e interpretación de la realidad tridimensional y de su representación cartográfica: 2D/3D, introducir al alumno en las técnicas de modelización 3D de terrenos e integrar nuevas tecnologías en la docencia universitaria contribuyendo al desarrollo técnico de la oferta de docencia virtual en la ULL. La metodología consiste en la realización de modelos digitales de terreno con elementos geomorfológicos establecidos previamente en 2D utilizando software de modelado 3D. Los modelos digitales terreno son un potente recurso utilizado en tareas de análisis topográfico para el reconocimiento de formas del terreno (Prasannakumar et al., 2011). Las tencnologías de visualización y representación tridimensional del territorio pueden tener un gran impacto en la investigación educativa sobre en el ámbito de las ciencias de la Tierra (Zhu, Pan and Gao, 2016). 


\section{TALLER DE MODELADO 3D}

En el taller participaron 38 alumnos de las titulaciones de Grado en Ingeniería Agrícola y del Medio Rural y de Grado en Ingeniería Civil, pertenecientes a la Escuela Politécnica Superior de Ingeniería de la Universidad de La Laguna, dentro de las actividades previstas en la planificación de asignaturas del Área de Ingeniería Cartográfica, Geodésica y Fotogrametría, del Departamento de Técnicas y Proyectos en Arquitectura e Ingeniería.

El software empleado fue el visor de la Infraestructura de Datos Espaciales de Canarias (Figura 1) (https://visor.grafcan.es/visorweb/) y la aplicación Sketch Up Sandbox (Figura 2).

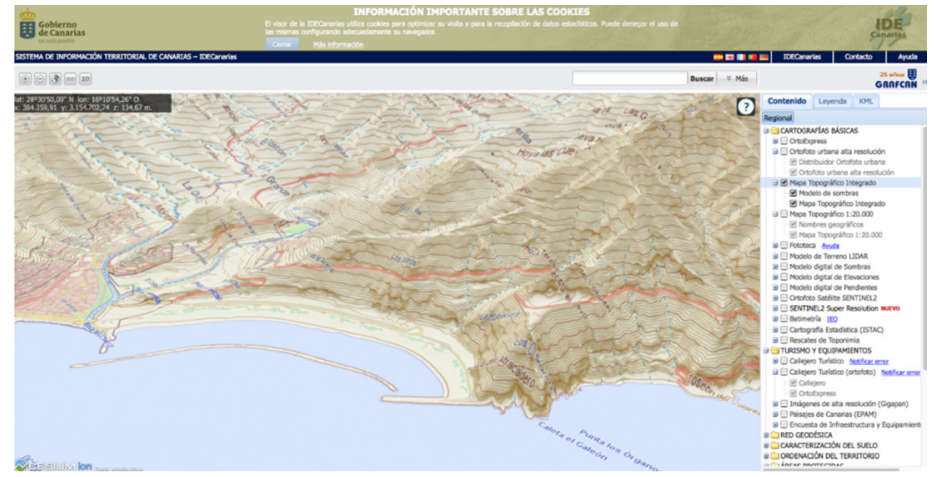

Figura 9. Visor de la Infraestructura de Datos Espaciales de Canarias.

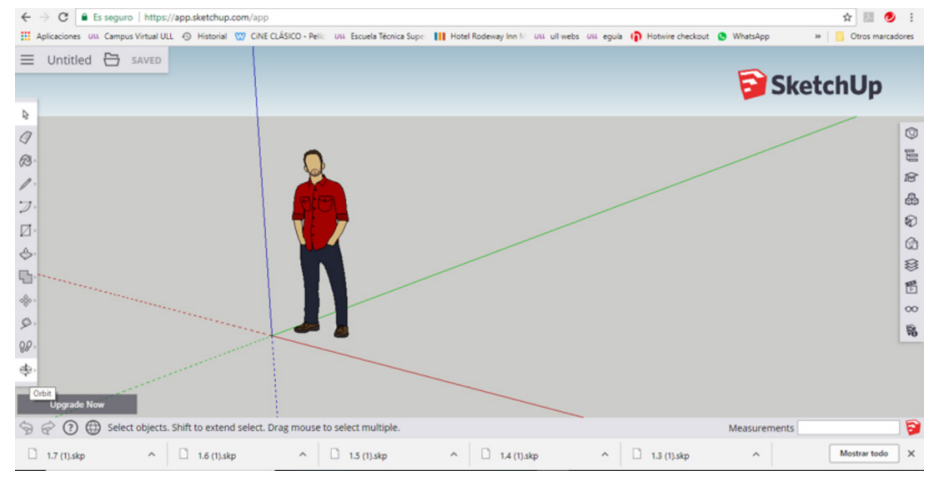

Figura 10. Interface Sketch Up Sandbox.

Antes y después de la actividad los alumnos realizaron el Topographic Map Asessment Test, del Research and Spatial Cognition Lab (RISC), Temple University (Jacovina et al., 2014).

En el taller se llevaron a cabo 2 actividades. Actividad 1: Modelar, con la aplicación SketchUp Sandbox, las siguientes formas del terreno: (1) una llanura, (2) una elevación, (3) una depresión, (4) una divisoria, 
(5) una vaguada, (6) un collado y, finalmente, (7) un terreno en el que figuren las formas del terreno 1,2,3,4,5 y 6 . Los estudiantes entregaron, en el aula virtual de la asignatura, un fichero por cada una de las 7 formas del terreno solicitadas. La Actividad 2 consitió en que, con el visor de la Infraestructura de Datos Espaciales de Canarias, loa estudiantes imprimeran imprimir un fichero PDF (usando la herramienta de impresión de la aplicación) con ejemplos de las formas del terreno 1, 2, 3, 4, 5 y 6 solicitadas en la actividad 1 . Se les solicitó a los participantes que los ficheros estuvieran impresos utilizando la doble pantalla que ofrece el visor de la Infraestructura de Datos Espaciales de Canarias, indicando con líneas las divisorias y las vaguadas, con un polígono las llanuras y con puntos las elevaciones, las depresiones y los collados. La entrega de la actividad 2 también se realizó utilizando el aula virtual de la asignatura.

La Figura 3 muestra ejemplos de la actividad 1 realizada por los estudiantes, donde se puede observar el modelado de una elevación realizado con la aplicación Skecth Up Sandbox (malla y renderizado):

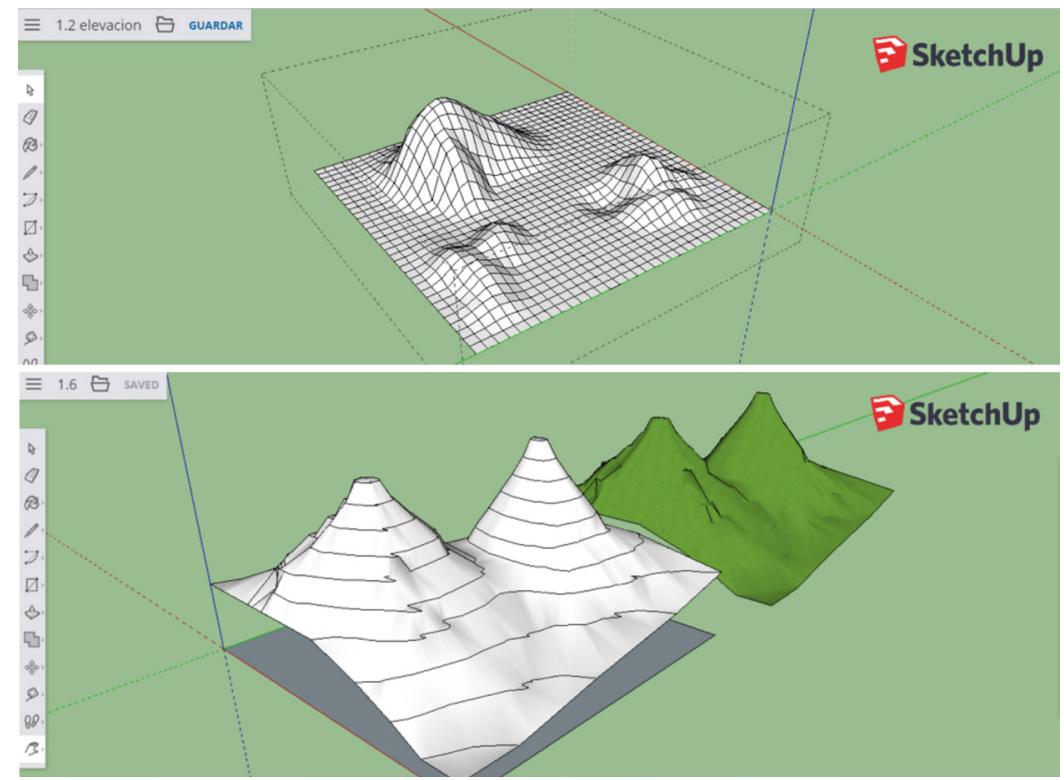

Figura 11. Modelado de una elevación (malla y renderizado).

Las figura 4 muestra la fusión de una imagen del terreno en 3D obtenido con la Infraestructura de Datos Espaciales de Canarias con curvas de nivel: Figura 12. Modelado de una elevación (IDE Canarias 3D y Sketch Up). 


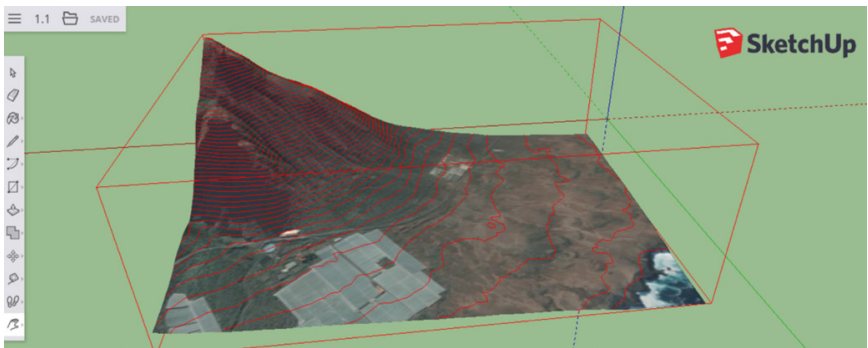

RESULTADOS

La Tabla 1 muestra el cuestionario y sus resultados. La fiabilidad de los cuestionarios se calculó utilizando el Alfa de Cronbach, obteniendo un valor 0.72 para el taller de SketchUp y 0.77. George y Mallery (2003) establecieron la siguiente escala para el coeficiente alfa de Cronbach:> 0.9 excelente; $>0.8$ bien; 0.7 aceptable; 0.6 cuestionable y $>0.5$ pobre.

\begin{tabular}{|c|c|c|c|}
\hline \multicolumn{4}{|c|}{ Tabla 1} \\
\hline \multicolumn{3}{|c|}{$\begin{array}{l}\text { Cuestionario de modelado 3D. Escala de Likert. } \\
\text { (1:totalmente en desacuerdo, 3: de acuerdo, 5: totalmente de acuerdo) }\end{array}$} & $\begin{array}{l}\text { Taller Mo- } \\
\text { delado 3D } \\
\text { Media(SD) }\end{array}$ \\
\hline \multirow{3}{*}{ 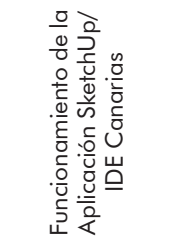 } & Q1 & $\begin{array}{l}\text { SketchUp Make es una buena herramienta } \\
\text { para el modelado 3D. }\end{array}$ & $\begin{array}{l}4.32 \\
(0.82)\end{array}$ \\
\hline & Q2 & $\begin{array}{l}\text { Las aplicaciones SketchUp Make e } \\
\text { IDE Canarias son estables, no se bloquean. }\end{array}$ & $\begin{array}{l}4.21 \\
(0.79)\end{array}$ \\
\hline & Q3 & $\begin{array}{l}\text { El Modedo 3D con SketchUp Make es } \\
\text { sencillo e intuitivo. }\end{array}$ & $\begin{array}{l}3.25 \\
(0.96)\end{array}$ \\
\hline \multirow{3}{*}{ 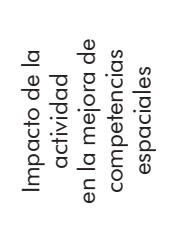 } & Q4 & $\begin{array}{l}\text { La actividad desarrollada en el taller ha } \\
\text { contribuido a mi alfabetización geográfica. }\end{array}$ & $\begin{array}{l}3.79 \\
(0.96)\end{array}$ \\
\hline & Q5 & $\begin{array}{l}\text { Creo que este tipo de actividades de modelado 3D pueden } \\
\text { desarrollar mi pensamiento geoespacial. }\end{array}$ & $\begin{array}{l}3.91 \\
(1.03)\end{array}$ \\
\hline & Q6 & $\begin{array}{l}\text { Creo que este tipo de actividades de modelado 3D } \\
\text { pueden desarrollar mi capacidad de interpretación del relieve. }\end{array}$ & $\begin{array}{l}4.45 \\
(0.96)\end{array}$ \\
\hline \multirow{3}{*}{ 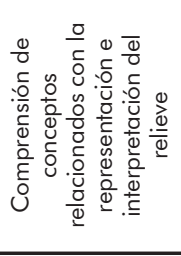 } & Q7 & $\begin{array}{l}\text { Este tipo de actividades de Modelado 3D del } \\
\text { terreno me ayuda a comprender mejor el } \\
\text { concepto de curva de nivel. }\end{array}$ & $\begin{array}{l}4.09 \\
(084)\end{array}$ \\
\hline & Q8 & $\begin{array}{l}\text { Este tipo de actividades de Modelado 3D del } \\
\text { terreno me ayuda a comprender mejor el } \\
\text { concepto de línea de maxima pendiente. } \\
\end{array}$ & $\begin{array}{l}4.43 \\
(0.81)\end{array}$ \\
\hline & Q9 & $\begin{array}{l}\text { Este tipo de actividades de Modelado 3D del } \\
\text { terreno me ayuda a comprender mejor los conceptos de perfil } \\
\text { longitudinal y transversal del terreno. }\end{array}$ & $\begin{array}{l}4.09 \\
(1.04)\end{array}$ \\
\hline \multirow{3}{*}{ 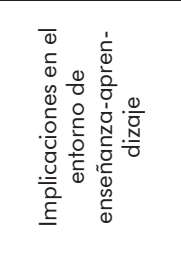 } & Q10 & $\begin{array}{c}\text { Este tipo de actividades de Modelado 3D del } \\
\text { terreno son un buen complemento a la enseñanza tradicional } \\
\text { de la Topografía y la Cartografía. }\end{array}$ & $\begin{array}{l}3.45 \\
(1.10)\end{array}$ \\
\hline & Q11 & $\begin{array}{l}\text { Este tipo de actividades de Modelado 3D del terreno son } \\
\text { relevantes en mi formación como Ingeniero }\end{array}$ & $\begin{array}{l}3.97 \\
(0.98)\end{array}$ \\
\hline & Q12 & $\begin{array}{l}\text { SketchUp y la IDE de Canarias son herramientas } \\
\text { apropiadas para llevar a cabo estrategias de } \\
\text { enseñanza en el ámbito de la Topografía y la Cartografía. }\end{array}$ & $\begin{array}{l}4.02 \\
(1.21)\end{array}$ \\
\hline
\end{tabular}




\section{CONCLUSIONES}

Las aplicaciones empleadas en el taller de modelado 3D (Skecth Up e Infraestructura de Datos Espaciales de Canarias) son una buena herramienta para el modelado 3D $(4,32)$. Su funcionamiento es estable, no se bloquea, lo que contribuye a que el estudiante se familiarice con estas tecnologías desde un principio y trabaje de forma continucada en la construcción de un modelo digital del terreno tridimensional $(4,21)$ a través de un interface con pocos comandos que permiten realizar esta tarea de un modo sencillo e intuitivo $(3,25)$. Las puntuaciones obtenidas en el apartado «Funcionamiento de la aplicación Sketch Up e IDE Canarias) son superiores a 3,24 sobre 5: estas aplicaciones son apropiadas para un primer contacto con las tecnologías de modelado 3D basado en información geoespacial.

Las activiades llevadas a cabo en el taller de modelado 3D han contribuido a mejorar la alfabetización geográfica de los participantes (3.79). Han empleado bases de datos geográficas con diferentes modos de visualización (mapa topográfico tradicional con curvas de nivel en 2D, modelos digitales de terreno procedentes de imágenes LiDAR, modelos digitales de pendientes, ortofotos...). Los participantes piensan que este tipo de actividades puede desarrollar su pensamiento geoespacial $(3,91)$ y su capacidad de interpretación del relieve cartográfico. Con valores superiores a 3,78 sobre 5 en el apartado del impacto de la actividad en el desarrollo de competencias espaciales, la opinión de los estudiantes sobre los efectos de esta actividad de modelado 3D es que favorece la mejora de competencias espaciales. Estas competencias espaciales están contempladas en los planes de estudio de Grados de Ingeniería adaptados al Espacio Europeo de Educación Superior.

Los participantes perciben que actividades de modelado 3D como las llevadas a cabo en la presente investigación facilitan la comprensión de conceptos relacionados con las técnicas cartográficas tradicionales de representación del terreno como las curvas de nivel $(4,09)$. Ayuda, a su vez, a la asimilación de conceptos ampliamente utilizados en Ingeniería relacionados con la representación del terreno como son la línea de máxima pendiente $(4,43)$ y perfil longitudinal y transversal $(4,09)$. Con valores todos por encima de 4,08 sobre 5, la percepción de los estudiantes sobre las actividades desarrolladas en el taller y su impacto en la comprensión de conceptos relacionados con la representación e interpretación del relieve $s$ alta.

Las activiades de modelado 3D son un buen complemento a la enseñanza tradicional de la Topografía y la Cartografía $(3,45)$. Los participantes consideran, además, que este tipo de actividades son relevantes dentro de los planes de formación de un estudiante de Ingeniería (3.97). Aplicacoines como Sketh Up e Infraestructura de Datos Espaciales de Canarias con apropiadas para planificar y llevar a cabo estrategias de 
enseñanza-aprendizaje basadas en tecnologías de modelado $(4,02)$. Los estudiantes peciben que este tipo de estrategias y/o metodologías docentes aportan valor en el entorno de enseñanza-aprendizaje, con puntuaciones superiores a 3,96 en todos los ítems de la subescala «implicaciones en el entorno de enseñanza-aprendizaje».

\section{REFERENCIAS BIBLIOGRÁFICAS}

Carbonell C. \& Bermejo L.A. (2017). Landscape interpretation with augmented reality and maps to improve spatial orientation skill. Journal Of Geography In Higher Education, 4(1), pp. 119-133. http://dx.DOI.org/10.1080/03098265.2016.1260530.

Carbonell, C., Mejías, M. A., Saorín, J. L., \& Contero, M. C. (2012). Spatial data infrastructure: Development of spatial abilities in the framework of european space for higher education. Boletín de la asociación de geógrafos españoles, 58(2012), pp. 157-175.

GeORge, D., \& MALLeRY, M. SPSS for Windows step by step: a simple guide and reference. Boston: Allyn \& Bacon, 2003.

Hegarty, M., Montello, D. R., Richardson, A. E., Ishikawa, T., \& Lovelace, K. (2006). Spatial abilities at different scales: Individual differences in aptitude-test performance and spatial-layout learning. Intelligence, 34(2), pp. 151-176. http://DOI. org/10.1016/j.intell.2005.09.005.

Jacovina, M., C. Ormand, T. F. Shipley, and Weisberg, S. 2014. Topographic Map Assessment. http://www.silccen ter.org/index.php/testsainstruments.

Linn, M. C., \& Petersen, A. C. (1985). Emergence and characterization of sex differences in spatial ability: A meta-analysis. Child Development, 56(6), pp. 1479-1498. DOI: 10.2307/1130467.

MAIER, P. H. (1998). Spatial geometry and spatial ability: How to make solid geometry solid. Proc., Annual Conf. of Didactics of Mathematics, E. Osnabrück, E. CohorsFresenborg, K. Reiss, G. Toener, and H. Weigand, eds., Gessellschaft für Didaktik der Mathematik, Munich, Germany, 63-75.

Prasannakumar, V., Shiny, Geetha, R. and VIJith, H. Applicability of SRTM data for landform characterisation and geomorphometry: a comparison with contour-derived parameters. International Journal of Digital. Earth 2011, 4, 387-401, doi:10.1080 /17538947.2010.514010.

ZHU, L., X. PAN and GAO, G. Assessing Place Location Knowledge Using a Virtual Globe. Journal of Geography, 2016, 112, 72-80, doi:10.1080/00221341.2015.1043930. 